\title{
Multiple Parietal Reach Regions in Humans: Cortical Representations for Visual and Proprioceptive Feedback during On-Line Reaching
}

\author{
Flavia Filimon, ${ }^{1,3}$ Jonathan D. Nelson, ${ }^{2,3,4}$ Ruey-Song Huang, ${ }^{1}$ and Martin I. Sereno ${ }^{1,5,6}$ \\ Departments of ${ }^{1}$ Cognitive Science and ${ }^{2}$ Computer Science and Engineering, University of California, San Diego, La Jolla, California 92093 , ${ }^{3}$ Max Planck \\ Institute for Human Development, 14195 Berlin, Germany, ${ }^{4}$ Computational Neurobiology Laboratory, Salk Institute for Biological Studies, La Jolla, \\ California 92037, ${ }^{5}$ School of Psychology, Birkbeck College, London WC1E 7HX, United Kingdom, and ${ }^{\circ}$ Birkbeck/University College London Neuroimaging \\ Centre, London WC1H 0AP, United Kingdom
}

Reaching toward a visual target involves at least two sources of information. One is the visual feedback from the hand as it approaches the target. Another is proprioception from the moving limb, which informs the brain of the location of the hand relative to the target even when the hand is not visible. Where these two sources of information are represented in the human brain is unknown. In the present study, we investigated the cortical representations for reaching with or without visual feedback from the moving hand, using functional magnetic resonance imaging. To identify reach-dominant areas, we compared reaching with saccades. Our results show that a reachdominant region in the anterior precuneus $(\mathrm{aPCu})$, extending into medial intraparietal sulcus, is equally active in visual and nonvisual reaching. A second region, at the superior end of the parieto-occipital sulcus (sPOS), is more active for visual than for nonvisual reaching. These results suggest that aPCu is a sensorimotor area whose sensory input is primarily proprioceptive, while sPOS is a visuomotor area that receives visual feedback during reaching. In addition to the precuneus, medial, anterior intraparietal, and superior parietal cortex were also activated during both visual and nonvisual reaching, with more anterior areas responding to hand movements only and more posterior areas responding to both hand and eye movements. Our results suggest that cortical networks for reaching are differentially activated depending on the sensory conditions during reaching. This indicates the involvement of multiple parietal reach regions in humans, rather than a single homogenous parietal reach region.

\section{Introduction}

The parietal lobe contains sensorimotor representations that use sensory input, e.g., vision, to guide interactions with the environment, e.g., reaching to a target. Whereas macaque neurophysiology has revealed parietal regions that use various sensory representations (visual, somatosensory) to guide multiple actions (eye, limb, head movements), the human homologs of those sensorimotor representations remain unclear (Culham et al., 2006), both in terms of effector specificity of the motor component (e.g., hand vs eye) and in terms of the sensory input driving the action (vision or somatosensation). For instance, both visual and proprioceptive feedback from the limb can guide on-line reaching. How these two sources of information map onto human parietal reach regions is unknown.

In macaques, several medial posterior parietal areas contain

\footnotetext{
Received July 8, 2008; revised Jan. 16, 2009; accepted Jan. 26, 2009.

This work was funded by National Science Foundation (NSF) Grant BCS 0224321 to M.I.S. and by NSF Integrative Graduate Education and Research Traineeship Program Grant DGE-0333451 to G. W. Cottrell and V. R. de Sa. We thank the University of California, San Diego fMRI Center, in particular, Giedrius T. Buracas, for technical support. We thank E. Todorov, G. Boynton, and J. Stiles for feedback on this manuscript and D. J. Hagler, Jr., for providing the original code for clustering and averaging.

Correspondence should be addressed to Flavia Filimon, Max Planck Institute for Human Development, KöniginLuise-Strasse 5, 14195 Berlin, Germany. E-mail: ffilimon@cogsci.ucsd.edu.

D01:10.1523/JNEUROSCI.3211-08.2009

Copyright $\odot 2009$ Society for Neuroscience $\quad$ 0270-6474/09/292961-11\$15.00/0
}

neurons that respond more during reaching than saccades, including area 5, V6A, and MIP, which partly overlaps the "parietal reach region” (PRR) (Kalaska, 1996; Fattori et al., 2001; Andersen and Buneo, 2002; Galletti et al., 2003).

Human functional neuroimaging has yielded inconclusive results on the human homolog(s) of macaque parietal reach regions, perhaps because most studies have used pointing preparation rather than actual reaching (Connolly et al., 2000, 2003; Astafiev et al., 2003; Fernandez-Ruiz et al., 2007; Hagler et al., 2007). Finger pointing is a much smaller movement than reaching, which may not recruit areas controlling the arm. In natural reaching, hands are not preshaped into a fist with the index finger extended, which is more typical of communicative gestures (Culham and Valyear, 2006; Culham et al., 2006). Reaching to point (with the index finger) (Prado et al., 2005), reaching to grasp (Grafton et al., 1996; Culham et al., 2008), and reaching to touch (Pellijeff et al., 2006; Levy et al., 2007; Culham et al., 2008) activate medial parietal cortex, although various locations and activation magnitudes (relative to saccades) have been reported. Importantly, various precuneus regions, including anterior precuneus $(\mathrm{aPCu})$ and superior parieto-occipital sulcus (sPOS), have been labeled "human PRR."

Recently, execution of visually guided reaching without touching, pointing, or grasping was shown to activate the entire superior extent of the precuneus, from the POS to the cingulate 
sulcus (Filimon et al., 2007). Does the precuneus map visual input from the hand, or proprioceptive feedback, during movement?

To investigate the medial parietal representations of visual versus proprioceptive feedback during reaching, we compared reaching with the hand visible and nonvisible. Whereas visual reaching involves both visual feedback and proprioception, nonvisual reaching requires online monitoring of proprioceptive feedback during the transport phase. This information is used to calculate where the hand is in space, relative to the target, without vision. Parietal reach-related regions exhibiting increased activation during visible compared with nonvisible reaches would reveal modulation from visual feedback. Parietal regions involved in on-line reaching that are not modulated by visual feedback would suggest proprioceptive input. Subjects used nondelayed (immediately executed), direct (withouta-mirror) reaching (involving the forearm and an open hand) toward external peripheral targets. To identify reachdominant areas, we also contrasted reaching with saccades. Given previous descriptions of multiple precuneus foci as human PRR, we examined how proprioceptive-only versus visual-andproprioceptive arm feedback modulates precuneus activations.

Results show that $\mathrm{aPCu}$ is activated equally whether or not the reaching hand is seen, whereas superior POS responds more during visual reaching. This suggests that $\mathrm{aPCu}$ is predominantly proprioceptive-motor and sPOS is predominantly visuomotor.

\section{Materials and Methods}

Subjects. Eight subjects (4 males and 4 females, age range 21-33) participated in this experiment. All subjects were neurologically intact and right handed, and had normal or correctedto-normal vision. All subjects gave written informed consent. The experimental procedures were approved by the University of California, San Diego Institutional Review Board.

Stimuli. Stimuli were fiber-optic targets mounted on a custom-made black screen (for a schematic, see Fig. 1a). The fixation point and each target consisted of the endpoint of a single optical fiber. The other end of each optical fiber (located outside the scanner room) was illuminated by a dim light-emitting diode (LED) controlled by a custom-made circuit board designed by R.-S.H. (see Huang and Sereno, 2008). Only one of five possible targets was illuminated at one time. The screen was mounted $\sim 35 \mathrm{~cm}$ in front of the subject, with the subject's head tilted forward for a direct view. The fiber-optic targets were arranged horizontally $\sim 7^{\circ}$ apart and $\sim 7^{\circ}$ below a fiber-optic fixation point, with the total display subtending $\sim 35^{\circ}$. All other sources of light inside the scanner room were turned off or covered up. The fiber-optic lights were dimmed such that a hand movement executed below each target was not visible to a subject inside the scanner. Each of the fiber-optic targets was turned on at random using a custom-made program on a Shuttle PC running Red Hat Linux.
Experimental task. Subjects were instructed to (1) reach to targets in the periphery while maintaining central fixation, (2) saccade, or (3) fixate. The targets were very dim fiber-optic points located horizontally below the fixation point (Fig. 1a). This was necessary to allow for reaching to just below each target, such that the hand would not obstruct the visual target or fixation point and thereby provide visual feedback as to its location. Subjects were instructed not to touch the screen. Reaching was done either in darkness, with no visual feedback from the hand (nonvisual direct reach), or in light, for which a bundle of optical fibers attached to the scanner bore above the subject's chest was turned on. A bright LED located inside the console room illuminated the bundle of optical fibers. The light illuminated the subject's hand during the reach, thus providing visual feedback (visual direct reach). Both kinds of reaching were executed directly toward the screen, in the same plane. Reaches were executed immediately, without delay. A pseudorandom block order of visual 
direct reach, nonvisual direct reach, saccade, and fixation trials was used. Each block of trials lasted $30 \mathrm{~s}$, with each reach trial lasting $4 \mathrm{~s}$, and each saccade trial lasting $2 \mathrm{~s}$. A $2 \mathrm{~s}$ auditory message at the beginning of the block indicated which condition was about to begin. Each functional run consisted of 20 blocks total.

Magnetic resonance image data acquisition. Magnetic resonance images were collected on a GE 3T scanner at the Center for fMRI, University of California, San Diego, using an eight-channel head coil. Functional images were collected using an echoplanar $\mathrm{T} 2^{*}$ gradient echo pulse sequence [ 32 contiguous axial slices, $3.44 \times 3.44 \times 4 \mathrm{~mm}$ voxel size, $64 \times$ 64 matrix, repetition time $(\mathrm{TR})=2000 \mathrm{~ms}, 300$ TRs (volumes) per functional scan, echo time $=30 \mathrm{~ms}$, flip angle $=90^{\circ}$, bandwidth $=62.5$ $\mathrm{kHz}$. The slice volume included the whole brain. The first four volumes in each scan series were discarded automatically, to allow the magnetization to reach a steady state. At least three functional scans (runs) were acquired per subject. In addition, a T1-weighted fast spoiled gradientecho (FSPGR) alignment scan $(1 \times 1 \times 1.3 \mathrm{~mm}$ voxel $)$ was collected in each scanning session to align the functional images to a previously obtained high-resolution $(1 \times 1 \times 1 \mathrm{~mm})$ T1-weighted FSPGR anatomical scan. To prevent head motion, each subject's head was stabilized using individual dental impressions (bite bars) supported by a four-ball-joint yoke. In addition, foam was packed around the head inside the head coil, and the upper arm was immobilized with foam pads.

Functional magnetic resonance imaging data analysis. Functional magnetic resonance imaging (fMRI) data were analyzed using FreeSurfer (Fischl et al., 1999a, Dale et al., 1999) and AFNI (Cox, 1996) (for detailed procedures, see Filimon et al., 2007). Each subject's cortical surface was reconstructed from the separately obtained high-resolution T1-weighted anatomical scan using FreeSurfer. Functional images were superimposed on the T1-weighted alignment scan collected during the functional session and then registered with the high-resolution anatomical scan from which the cortical surface was reconstructed. This allowed functional activations to be painted onto each subject's reconstructed cortical surface. Functional runs were concatenated using AFNI's 3dTcat function. Slice-timing correction was implemented using AFNI's tshift function. Motion correction was implemented using AFNI's volreg (volume registration) function, registering images to the middle TR of the middle functional run. In five subjects there was a small amount of residual whole-head motion resulting in "spikes" in the raw signal time series which could not be corrected during volume registration (volreg). The TRs affected by head motion artifact were removed from the raw data using AFNI's censor function. On average, $4.22 \%$ of reach TRs and $4.24 \%$ of saccade TRs were excluded from those five subjects' data to remove motion artifact. The percentage of TRs (brain volumes) removed per reach, saccade, and rest (fixation) conditions to eliminate motion artifact, were as follows: subject 1: $1.83 \%$ (reach), $1 \%$ (saccade), $1.67 \%$ (rest); subject 2: 3.78\% (reach), 2.22\% (saccade), 9.33\% (rest); subject 3: $7.67 \%$ (reach), $1.67 \%$ (saccade), $7.67 \%$ (rest); subject 4: $1 \%$ (reach), $0 \%$ (saccade), 2.67\% (rest); subject 5: 6.83\% (reach), 16.33\% (saccade), $10 \%$ (rest). Subject 5 was the only subject with more head motion during the saccade condition compared with the reach condition. Since the activation pattern for each condition was the same as in other subjects even after removing the noisy TRs, subject 5 was still included in group analyses. The pattern of activation was not different between these five subjects and the remaining subjects, suggesting that motion artifact was successfully removed.

Blood oxygenation level-dependent (BOLD) responses to each experimental condition were analyzed using AFNI's 3dDeconvolve (Cox, 1996; Ward, 2000) general linear model (multiple regression), with a regressor of interest for each condition. A quadratic polynomial was used to fit the baseline. The 6 motion parameters obtained from volume registration were included as regressors in the baseline model to account for variance due to motion. Hemodynamic responses were modeled at six different lag times (1, 2, 3, 4, 5, and 6 TRs). Correlation coefficients and $F$-statistics were generated for the area under the hemodynamic response function. Visual reach, nonvisual reach, and saccade were each contrasted with the fixation baseline, as well as with each other (visual reach vs saccade; nonvisual reach vs saccade, visual reach vs nonvisual reach).

Group analysis. Each subject's reconstructed cortical surface was sphered and registered to an average spherical surface atlas in FreeSurfer using a best-fit sulcal alignment (Fischl et al., 1999b). Each subject's coefficients and $F$-statistics were interpolated onto the average spherical surface after using 16 steps of surface-based smoothing, equivalent to a full-width, half-max (FWHM) Gaussian filter of $\sim 5 \mathrm{~mm}$ (Hagler et al., 2006). Activations were averaged across subjects in this spherical coordinate system. A one-way, repeated-measures ANOVA was performed for each voxel on the whole brain using AFNI's mixed-effects, two-factor 3dANOVA2, to generate means and $F$-statistics of activations for each condition versus baseline (visual direct reach, nonvisual direct reach, and saccade), and for pairwise comparisons. Condition was a fixed effect (with three levels), while subject was a random effect. Averaged activations and F-statistics were resampled back onto an individual subject's inflated cortical surface for display purposes. To correct for multiple comparisons, we used surface-based clustering (Hagler et al., 2006). Clusters of contiguous vertices were identified for $t$-statistics thresholded at $p<0.05$ for the group-averaged data and $p<0.001$ for individual subjects.

Region of interest analysis. We defined parietal regions of interest (ROIs) within each subject based on both anatomical and functional criteria. ROIs consisted of contiguous voxels located on the medial parietal surface, between the POS and the cingulate sulcus. Within the medial parietal surface, voxels were identified that were active in the visual reach versus baseline comparison, since (1) the aim was to identify medial parietal regions activated by reaching, and compare reach-related and saccade-related activations within such areas; (2) visual reaching yielded the greatest extent of reach-related activations; and (3) a separate aim was to see whether nonvisual reach activations and visual reach activations differed within such areas (e.g., to identify whether nonvisual reach activations might be weaker or less extended compared with visual reach activations). Only contiguous voxels significant within each subject at $p<0.005$, corrected, were included. This yielded an anterior precuneus region of interest. Each subject's data set was divided in two, with ROIs identified in one half of the data, and percent signal change and time series analyses performed on the other half.

An additional region of interest was identified based on the pattern of activations for visual and nonvisual reaching compared with saccades around the POS. The region was situated in-between the superior end of the POS and the subparietal sulcus. These anatomical criteria were used in addition to the same functional criteria as above to define an sPOS region of interest in half the data, with calculations performed as noted above on the other half of the data. The POS ROI became disconnected from the anterior precuneus ROI at higher thresholds ( $p<0.005$, corrected), suggesting the presence of two separate areas. ROIs were saved as surface patches in FreeSurfer. Voxels within each patch were normalized by their mean intensity and averaged within the ROI using MATLAB. Percent signal change coefficients were calculated for each condition from the normalized time series within the ROI.

\section{Results}

Figure 1 shows the experimental setup for the experiment. Note that "visually guided reaching" could refer either to reaching guided by a visual target or to reaching with the hand visible. Here, we refer to reaching with the hand visible as "visual reaching" and to reaching with the hand nonvisible as "nonvisual reaching." Moreover, since reaches were executed directly toward the peripheral visual targets, we refer to this as "direct" reaching (as opposed to, e.g., indirect reaches toward hidden targets). In the present experiment, subjects executed visual and nonvisual direct reaches or saccades to peripheral visual targets in front of them. Subjects reached from below toward the dim targets, without moving the hand on top of the targets, so that no visual feedback could be gained from obstructing the light source. All subjects confirmed that they could not see their hand in the nonvisual condition. During the visual reach condition, a bright fiber-optic light taped to the ceiling of the scanner bore illuminated the subject's moving hand. This allowed for a direct com- 


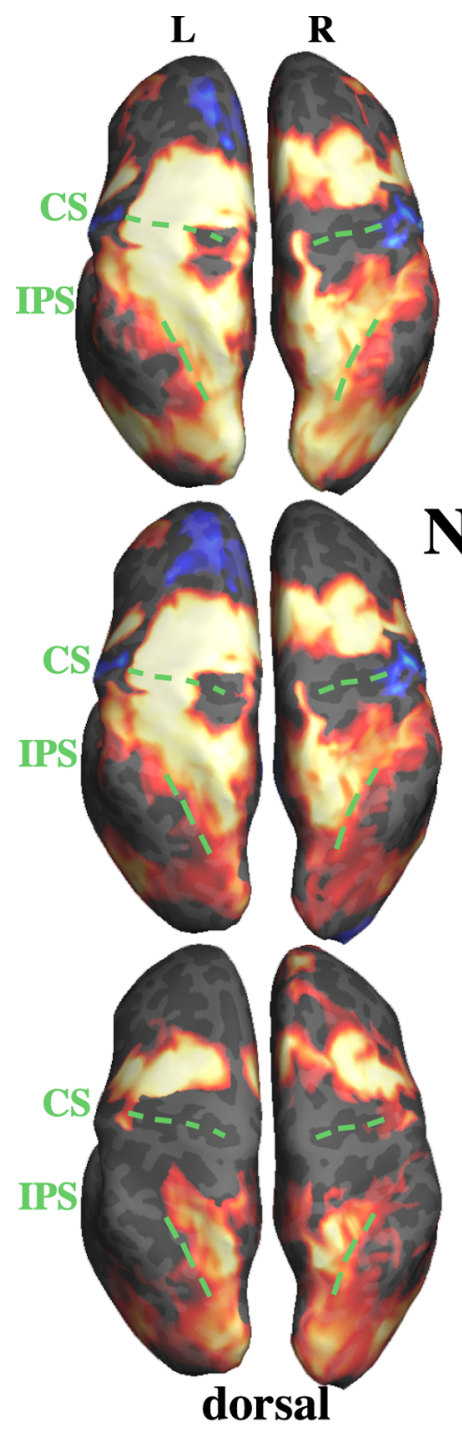

\section{VISUAL DIRECT REACH}

$\mathbf{L}$

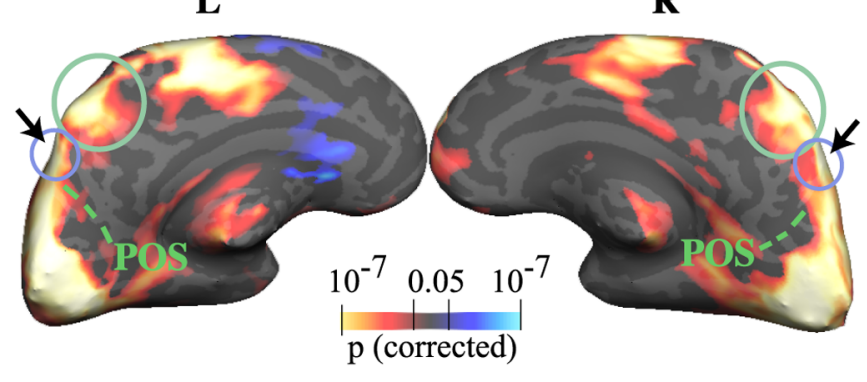

$\mathbf{L}$

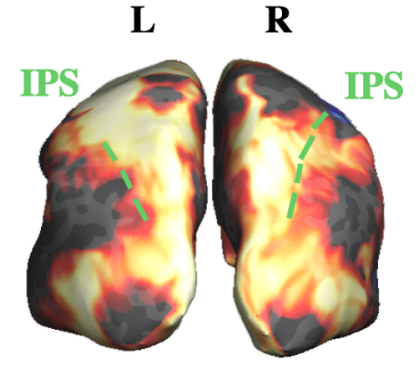

NON-VISUAL DIRECT REACH

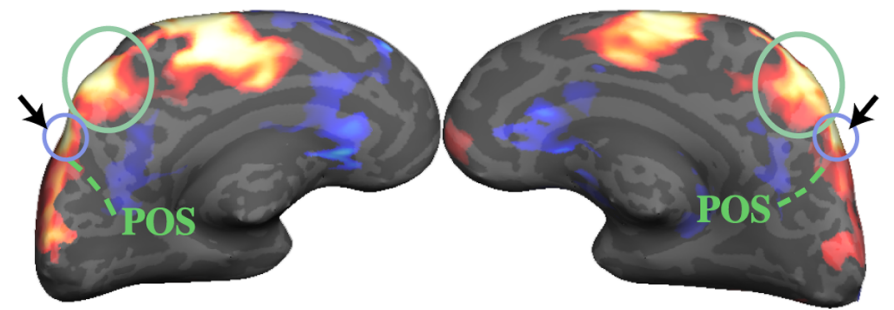

\section{SACCADE}

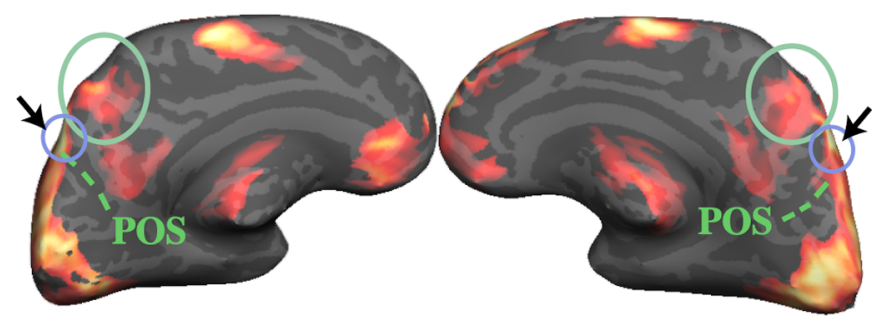

medial
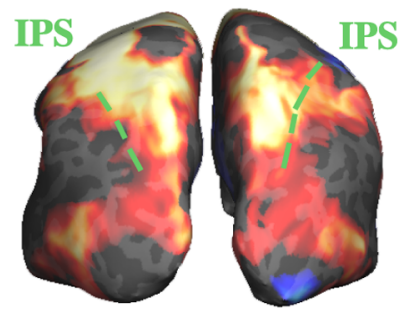

IPS

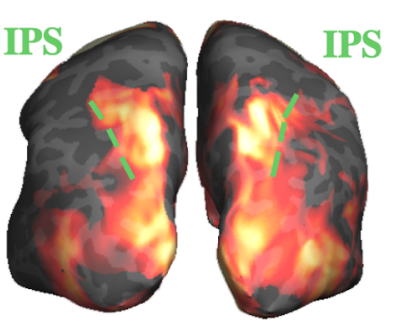

posterior

Figure 2. BOLD activations for visual direct reaching, nonvisual direct reaching, and saccades. Group surface-averaged activations ( $n=8)$ for visual and nonvisual direct reaching and saccades, versus baseline, are shown on dorsal, medial, and posterior views of the left and right inflated cortical hemispheres. Note the similarity in activation between visual and nonvisual direct reaching in the green-circled anterior precuneus region (medial view). Saccade activations in the same anterior precuneus ROI are much weaker. Visual direct reaching also activates the sPOS more strongly than saccades or nonvisual direct reaching (see arrows and small purple-blue circle). Sulci are marked with a dashed green line. CS, Central sulcus; L, left; R, right.

parison of visual direct reaching with nonvisual direct reaching to the same targets, in the same reach plane. Central fixation was maintained during both visual and nonvisual reaching. All reaches were executed immediately, without a delay.

Figure 2 shows group-average activations $(p<0.05$, corrected) for visual direct reaching, nonvisual direct reaching, and saccades, versus fixation. Both kinds of reaching elicited a very similar pattern of frontoparietal activations compared with baseline, including dorsal and ventral premotor, supplementary motor, primary somatomotor, cingulate, intraparietal, superior parietal, and medial parietal cortical activations. Activations were bilateral but stronger in the left hemisphere, with primary somatomotor activations entirely left lateralized, as expected for right-handed subjects moving their right hand only.

As shown in Figure 2, both visual and nonvisual reaching strongly activated the precuneus (medial parietal cortex) bilaterally, from the posterior end of the cingulate sulcus through to the POS, continuing into the cuneus. The superior aspect of the POS was activated more strongly by visual reaching than by nonvisual reaching.
Saccades activated a frontoparietal network of brain regions that partly overlapped with reaching activations (Fig. 2, bottom row). Saccade activations were bilateral and included the frontal and supplementary eye fields (superior frontal sulcus/gyrus), the inferior precentral sulcus/gyrus, the intraparietal sulcus (IPS) and superior parietal gyrus (parietal eye fields), and the occipital cortex, including V1, the cuneus, lingual gyrus, and superior occipital gyrus. The precuneus was only weakly activated by saccades, bilaterally.

Both visual reaching and saccades strongly activated area V1 (calcarine sulcus), the lingual gyrus, precuneus, and the lateral and superior occipital gyri, due to visual stimulation from the hand during reaching, and due to foveation of targets during saccades (Fig. 2, top and bottom rows). Occipital activations during nonvisual reaching were greatly reduced compared with visual reaching or saccades, and did not extend into the calcarine sulcus or lingual gyrus (Fig. 2, middle row). Some occipital activation during nonvisual reaching was expected, as the peripheral fiber-optic targets provided visual stimulation even when the hand did not. A posterior region of the lateral occipital gyrus, 
including MT + (Fig. 2, dorsal and posterior views) was activated during nonvisual reaching as well as during visual reaching, consistent with tactile input to parts of MT+ (MST) (Beauchamp et al., 2007); part of this region may also represent the extrastriate body area, which responds to hand movement (Astafiev et al., 2004).

To identify reach-dominant areas, we subtracted saccaderelated activations from visual direct and nonvisual direct reaching activations (Fig. $3 a$, top and middle rows).

Our hypothesis was that a possible human homolog of the macaque parietal reach region, located on the medial surface of the parietal lobe, would also be more active during visual and nonvisual reaching than during saccades. Indeed, visual direct reaching activated the anterior part of the precuneus (circled in green) and the superior POS (circled in purple-blue) significantly more strongly than saccades, bilaterally (Fig. $3 a$, top row) $(p<$ 0.05 , corrected). Nonvisual direct reaching also activated the anterior part of the precuneus significantly more than saccades, but showed no difference in activation in the superior POS, bilaterally (Fig. $3 a$, middle row, central panel). Analysis of more stringent thresholds ( $p<0.001$; data not shown) revealed that anterior precuneus activations for nonvisual reaching versus saccades were stronger in the left hemisphere than the right; i.e., contralateral to the moving hand.

In addition to the anterior precuneus, other areas more strongly activated by both visual reaching and nonvisual reaching than by saccades included the anterior intraparietal sulcus (putative AIP), the supramarginal gyrus, medial intraparietal sulcus (putative MIP), and superior parietal gyrus in the posterior parietal lobe, as well as dorsal premotor cortex, primary somatosensory and primary motor cortex (precentral and postcentral gyri), and supplementary motor cortex (Fig. $3 a$, top and middle rows), with activations stronger or lateralized to the left hemisphere. Visual reaching also activated the occipital lobe and middle occipital cortex $(\mathrm{MT}+)$ more than saccades bilaterally. Saccades activated the left occipital pole and right occipital lobe more than nonvisual reaching, confirming that subjects did not have visual input from the hand during nonvisual reaching and did not foveate the targets during reaching. Saccades activated the left and right inferior central sulcus (eye representation) more than either type of reaching.

To identify parietal areas modulated by visual feedback from the hand during reaching, we compared visual reaching with nonvisual reaching (Fig. $3 a$, bottom row). This comparison revealed significantly stronger activations ( $p<0.05$, corrected) for visual reaching than nonvisual reaching around the sPOS, at the parieto-occipital junction, bilaterally. In contrast, the anterior precuneus and the rest of the parietal lobe did not differ significantly in activation between visual and nonvisual reaching. To confirm that our statistical correction was not overly stringent, we also compared visual and nonvisual reaching activations at the uncorrected 0.05 level, which still yielded no difference in the anterior precuneus. The sPOS activation also extended more anteriorly into the posteromedial aspect of the IPS; however, this anterior activation did not survive $p<0.01$. Since that region was activated equally for saccades when comparing both visual reach versus saccades and nonvisual reach versus saccades, it likely represents an effector-independent visual representation of visual stimuli (whether a hand or other stimulus).

In addition to sPOS, visual and nonvisual reaching activations (Fig. $3 a$, bottom row) differed in the occipital lobe, with the cuneus, calcarine sulcus, lingual gyrus, and superior and lateral occipital gyri (including MT +) being significantly more active dur- ing visual direct reaching than during nonvisual direct reaching ( $p<0.05$, corrected). This is consistent with the greater visual stimulation provided by the visual reach than the nonvisual reach.

Figure $3 a$ thus suggests that on the medial parietal wall, two areas contribute differentially to reaching: the anterior precuneus participates equally in visual and nonvisual reaching. We denote this region as the anterior precuneus reach region, or " $\mathrm{aPCu}$ " in short. In contrast, an area at the superior aspect of the parietooccipital sulcus, or sPOS, was more strongly activated by visual reaching than by either saccades or nonvisual reaching.

Figure $3 b$ shows the time course of activity and percent signal change in the left anterior precuneus across all three conditions. Percent signal change in BOLD signals for visual and nonvisual reaching in aPCu did not differ significantly $\left(t_{(7)}=0.44\right.$, n.s.). The magnitude of BOLD signal change in aPCu was significantly less for saccades than either for visual reaching $\left(t_{(7)}=6.98\right.$, twotailed $p=0.0004$ ) or for nonvisual reaching $\left(t_{(7)}=3.61\right.$, twotailed $p=0.01$ ).

Figure $3 c$ shows the percent signal change in the left sPOS for visual and nonvisual reaching and for saccades. Percent signal change in BOLD signals in the left sPOS was significantly greater for visual reaching than for nonvisual reaching $\left(t_{(7)}=3.34\right.$, twotailed $p=0.01)$ and than for saccades $\left(t_{(7)}=3.71\right.$, two-tailed $p=$ $0.004)$. Nonvisual reaching and saccade percent signal change did not differ in the left $\operatorname{sPOS}\left(t_{(7)}=-1.41\right.$, n.s.). This pattern is consistent with sPOS having a largely visuomotor role, and $\mathrm{aPCu}$ a predominantly proprioceptive-motor role, in reaching. Table 1 shows the average $x, y, z$ coordinates for the two ROIs.

To more closely examine the difference between the various parietal areas activated more strongly by both kinds of reaching than by saccades, and their relationship to the anterior precuneus, we implemented a conjunction analysis in AFNI. As can be seen from Figure 2, the aPCu is strongly activated by both visual and nonvisual reaching, and is weakly (but significantly, at $p<$ 0.05 ) activated by saccades. Figure $3, a$ and $b$, showed that aPCu is equally activated by visual and nonvisual reaching. To search for any other areas that behave similarly, we used a whole-brain conjunction analysis to identify voxels satisfying of all of these criteria: (1) the voxel is activated by visual reach, nonvisual reach, and saccade, above baseline at $p<0.05$; AND (2) the voxel is significantly more active in both visual reach versus saccade and nonvisual reach versus saccade $(p<0.05)$; i.e., visual reach - saccade $>0$; AND nonvisual reach - saccade $>0$; AND (3) visual reach - nonvisual reach $=0(p>0.05$, n.s. $)$.

Figure $4 a$ shows the result of this conjunction on an inflated left hemisphere (contralateral to the moving hand), with voxels satisfying all three criteria shown in yellow. As expected, an area located in the anterior precuneus (medial parietal cortex) and overlapping with the aPCu activations shown in Figures 2 and $3 a$, satisfies these criteria. Interestingly, the anterior precuneus activations are joined to a posterior parietal area in the medial intraparietal sulcus (medial IPS, or mIPS), which extends to the superior (dorsal) aspect of posterior parietal cortex. This area might correspond to macaque MIP, which has also been shown to be involved in reaching in macaques. In addition to $\mathrm{aPCu}$ and medial IPS, dorsal premotor cortex (superior frontal gyrus) and the supplementary motor cortex (SMA) also satisfy these criteria. This is expected, since parietofrontal circuits underlie reaching in the macaque, with both parietal and premotor areas contributing. Thus, it appears that within posterior parietal cortex, only medial IPS and aPCu participate in all three conditions, with greater activation to visual and nonvisual reaching than to sac- 


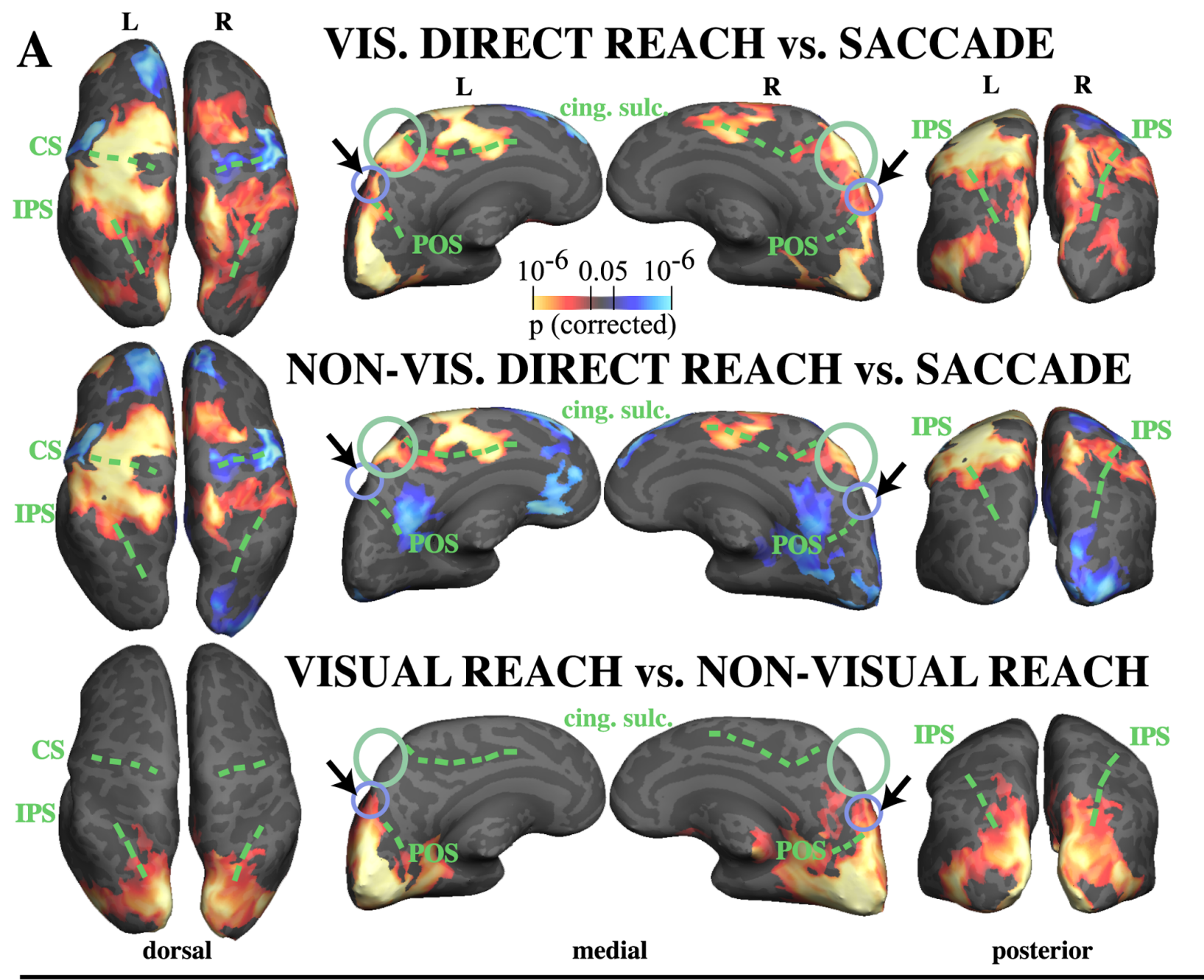

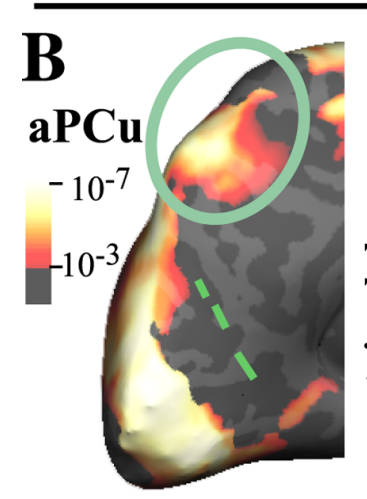

left medial
Average percent signal change over time in left aPCu

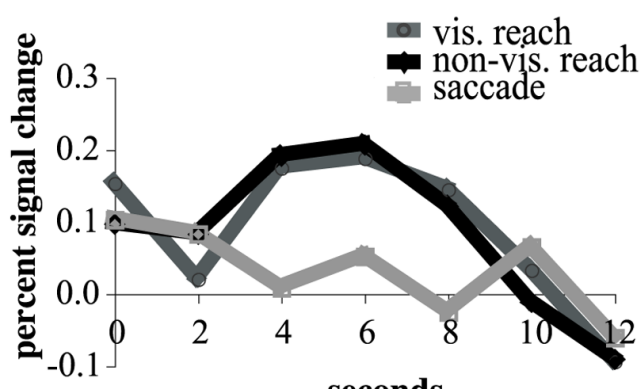

seconds
Percent signal change by condition in left aPCu

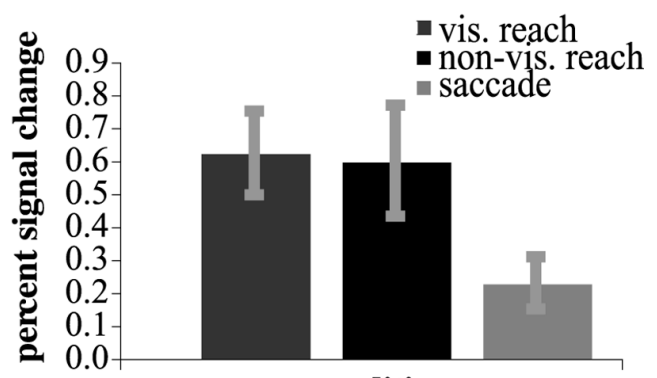

condition

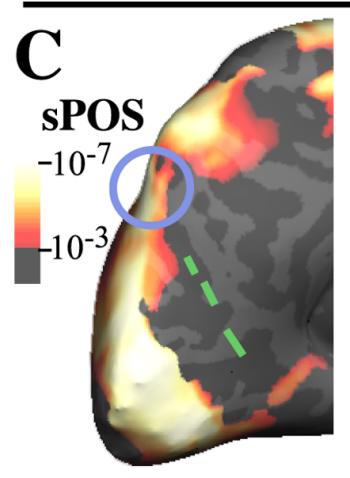

left medial
Average percent signal change over time in left superior POS

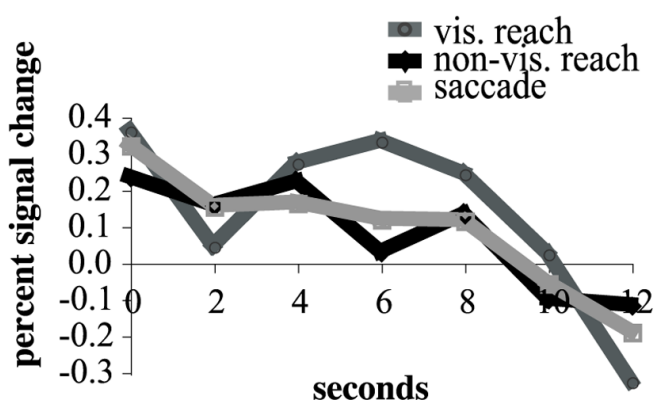

Percent signal change by condition in left superior POS

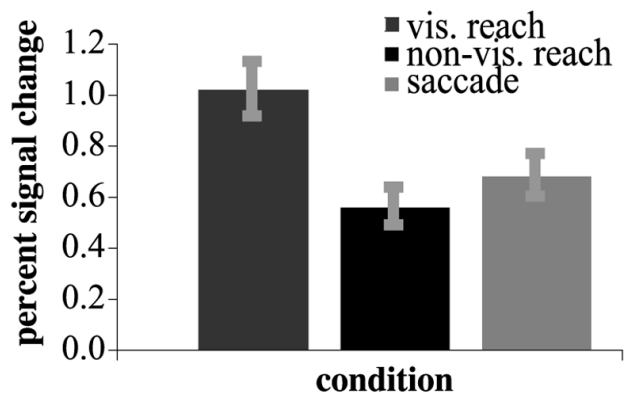


Table 1. Average MNI-space coordinates (in millimeters) for peak activations in the aPCu and superior POS regions of interest

\begin{tabular}{llrlll}
\hline Contrast & ROl & $x$ & $y$ & $z$ & No. of subjects \\
\hline Vis. reach > saccade & Left aPCu & $-9 \pm 1.7$ & $-54 \pm 3.1$ & $60 \pm 1.2$ & 7 \\
& Right aPCu & $11 \pm 0.9$ & $-54 \pm 2.4$ & $58 \pm 2.1$ & 7 \\
Non-vis. reach > saccade & Left aPCu & $-11 \pm 1.3$ & $-58 \pm 3.0$ & $61 \pm 1.8$ & 7 \\
& Right aPCu & $12 \pm 1.2$ & $-57 \pm 2.6$ & $60 \pm 1.8$ & 7 \\
Vis. reach > non-vis. reach & Left sPOS & $-9 \pm 1.9$ & $-77 \pm 2.1$ & $46 \pm 1.7$ & 7 \\
& Right sPOS & $13 \pm 1.7$ & $-78 \pm 1.7$ & 45 \\
\hline
\end{tabular}

The SEM ( \pm ) is indicated to the right of each $x, y$, and $z$ coordinate. One of the eight subjects had anomalous ( $>30 \mathrm{~mm}$ different) MNI coordinates and was thus excluded. All activations were at $p<0.001$ (corrected).

cades, and equal involvement in visual and nonvisual reaching. Notice the dip in the V-like activation pattern: the bottom of the $V$ lies on the crest of the inflated surface and thereby separates the medial (precuneus) activation from the dorsal (mIPS) activation (Fig. 4a).

Figure $4 b$ shows multiple conjunctions overlaid on the inflated left hemisphere. In green, only voxels are shown that are strongly activated by both visual and nonvisual reaching $(p<$ 0.01 ) but not by saccades. As can be seen in Figure $4 b$, parietal voxels activated only by reaching and not by saccades are anterior as well as lateral to the aPCu and mIPS activations identified by the conjunction in Figure $4 a$. From a medial view (Fig. $4 b$, middle panel), most of these voxels fall inside the posterior end of the cingulate sulcus, extending dorsally onto the very anterior aspect of the superior parietal gyrus. This location is consistent with what in the macaque would be called area 5 (Cavada, 2001), which mostly responds during hand movements but not during saccades, consistent with our analysis. The more anterior IPS activations (green) lateral of the yellow cluster might correspond (at least in anatomical location) to macaque areas 7a and AIP, also involved in hand movements (Andersen and Buneo, 2002). Voxels more anterior than the anterior IPS fall inside the primary somatosensory cortex (postcentral gyrus).

Voxels more active during visual reach compared with nonvisual reach are overlaid in light blue (Fig. $4 b$ ), showing that only areas located more posteriorly, at the parieto-occipital junction (sPOS), are modulated by vision during reaching.

Figure $4 c$ shows a conjunction between activations for all three conditions, i.e., voxels activated significantly by visual reaching, nonvisual reaching, and saccades, at $p<0.05$ (pale yellow) and $p<0.01$ (red). This figure shows that as the threshold is raised, the medial activation ( $\mathrm{aPCu}$ ) becomes disconnected from the more dorsal mIPS activation, suggesting that although coacti-

\footnotetext{
$\leftarrow$

Figure 3. Contrasts between activations for visual direct reaching and saccades, nonvisual direct reaching and saccades, and visual and nonvisual direct reaching. $A$, Contrast activations. Red to yellow indicates greater activation for the condition subtracted from; blue to light blue indicates greater activation for the subtracted condition. Both kinds of reaching activate the aPCu more than saccades. Note the lack of difference between visual and nonvisual direct reaching in aPCu, circled in green (bottom row, medial view). The superior POS is indicated with a black arrow and a purple-blue circle. Note the increased activation for visual direct reaching compared with nonvisual direct reaching in the SPOS region. $\boldsymbol{B}$, Percent signal change time course (left) and magnitude (right) in left aPCu for visual direct reaching, nonvisual direct reaching, and saccades, compared with baseline. Visual and nonvisual direct reaching activations are not significantly different in aPCu and exhibit a similar time course. Both kinds of reaching were significantly different from saccades in left aPCu ( $p<0.05$, corrected). C, Percent signal change time course (left) and magnitude (right) in the left superior POS for visual and nonvisual reaching, and saccades. Visual reaching activates the SPOS more than either nonvisual reaching $(p=0.01$ ) or saccades $(p=0.004)$. Error bars in $B$ and $C$ indicate the SEM. Bar graphs on the right represent the sum of percent signal change values at each time point shown in the curves on the left, i.e., over $0-6$ TRs. Activations to the left of graphs show visual direct reach versus baseline ( $p<0.001$, corrected). cing. sulc., Cingulate sulcus; $C$, central sulcus; $L$, left; $R$, right; vis., visual.
}

vated in the conjunction mask in Figure $4 a$, these could be two functionally distinct areas.

Our results thus show that (1) the precuneus has functionally distinct subregions. Specifically, the anterior precuneus is not modulated by sight of the hand during reaching, suggesting proprioceptive input, whereas the sPOS is modulated by sight of the hand; (2) the anterior precuneus is coactivated with medial IPS, although the two areas become disconnected at higher thresholds, implying some functional separation; and (3) as seen in Figure $4 b$, there is a gradual transition in functional characteristics from anterior to posterior parts of the posterior parietal lobule. This transition is from (i) reach-only areas (in green), which do not respond to saccades, in the very anterior parts of the posterior parietal lobule and at the posterior end of the cingulate sulcus, to (ii) both-reach-and-saccade areas (in yellow) in medial IPS and anterior precuneus, which respond more strongly and equally to both reaches compared with saccades, showing no visual modulation, to (iii) more posterior reach areas modulated by vision of the hand and saccades (in blue, sPOS).

Thus, whereas previous fMRI studies in humans have labeled various parts of the precuneus "human PRR," our current analysis shows that in fact the anterior part of the precuneus responds equally during visual and nonvisual reaches, and more weakly during saccades, as does medial IPS. In contrast, the more posterior sPOS area is modulated by vision of the hand during the reach. Our results thus argue for multiple parietal reach regions, with different roles in visual as opposed to proprioceptively guided reaching, rather than a "single parietal reach region."

Figure 5 shows contrast activations for visual reach versus saccade, nonvisual reach versus saccade, and visual versus nonvisual reach ( $p<0.0001$, corrected) from three representative subjects. Every subject showed significantly greater activation in aPCu for visual and nonvisual reaching compared with saccades, bilaterally. Figure 5 also shows greater sPOS activation for visual versus nonvisual reaching; this pattern was true in all but one subject.

Supplemental data from a separate experiment on imagined, observed, and executed visual reaching (see supplemental Fig. S1, available at www.jneurosci.org as supplemental material) show that both sPOS and aPCu are activated by reaching imagery alone, when no visual feedback or somatosensory input from the hand are available. Thus, sPOS and aPCu are not purely visual or purely somatosensory, respectively.

We also performed two additional experiments. In the first experiment, subjects saccaded or reached to visual targets displayed around the fixation cross in both the lower and upper visual field, with the hand in sight (see supplemental Fig. S2, available at www.jneurosci.org as supplemental material). In the second experiment, subjects saw the same visual targets as in the first additional experiment, except that reaching was performed out of sight on a hidden plate to the right of the subject, with no visual feedback from the hand (see supplemental Fig. S3, avail- 


\section{(Left hemisphere) CONJUNCTION ANALYSES}
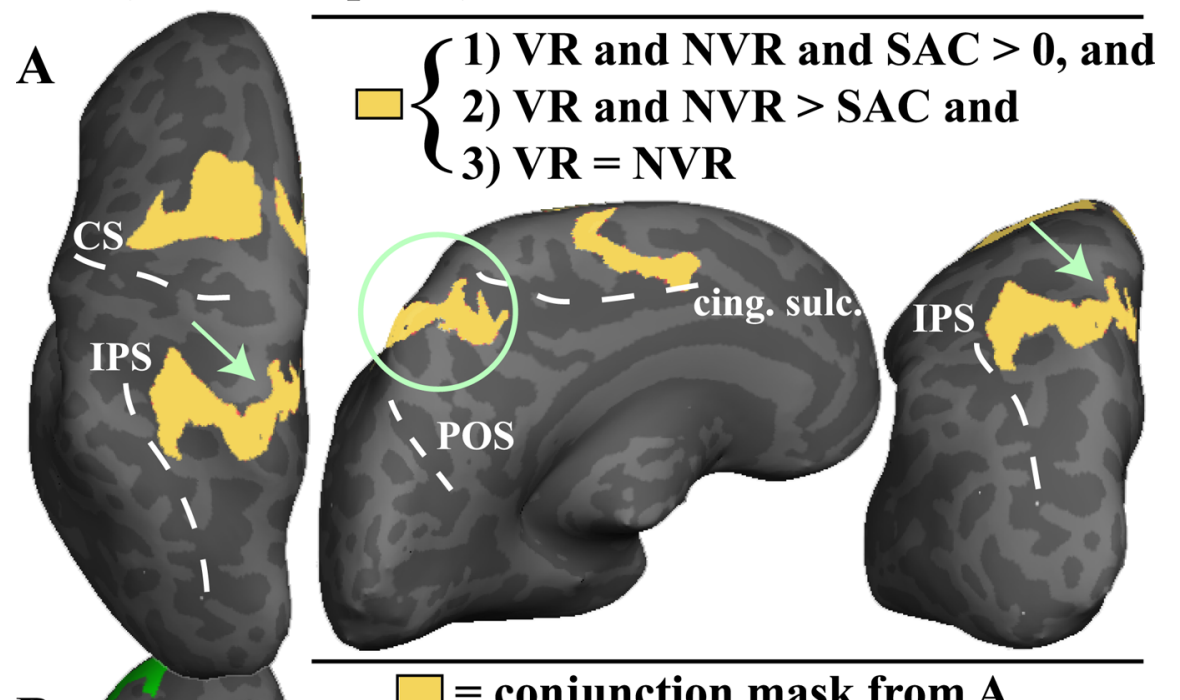

B

$\square=$ conjunction mask from $A$

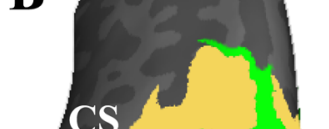

$\square=V R$ and NVR $>0$ and NOT SAC $\square=\mathbf{V R}>\mathbf{N V R}$

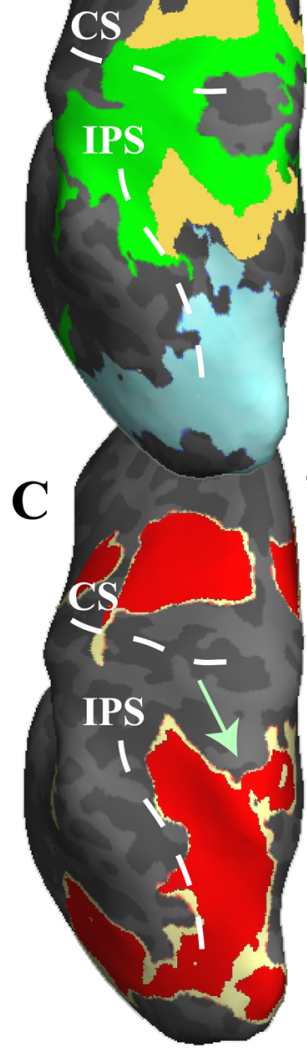

dorsal
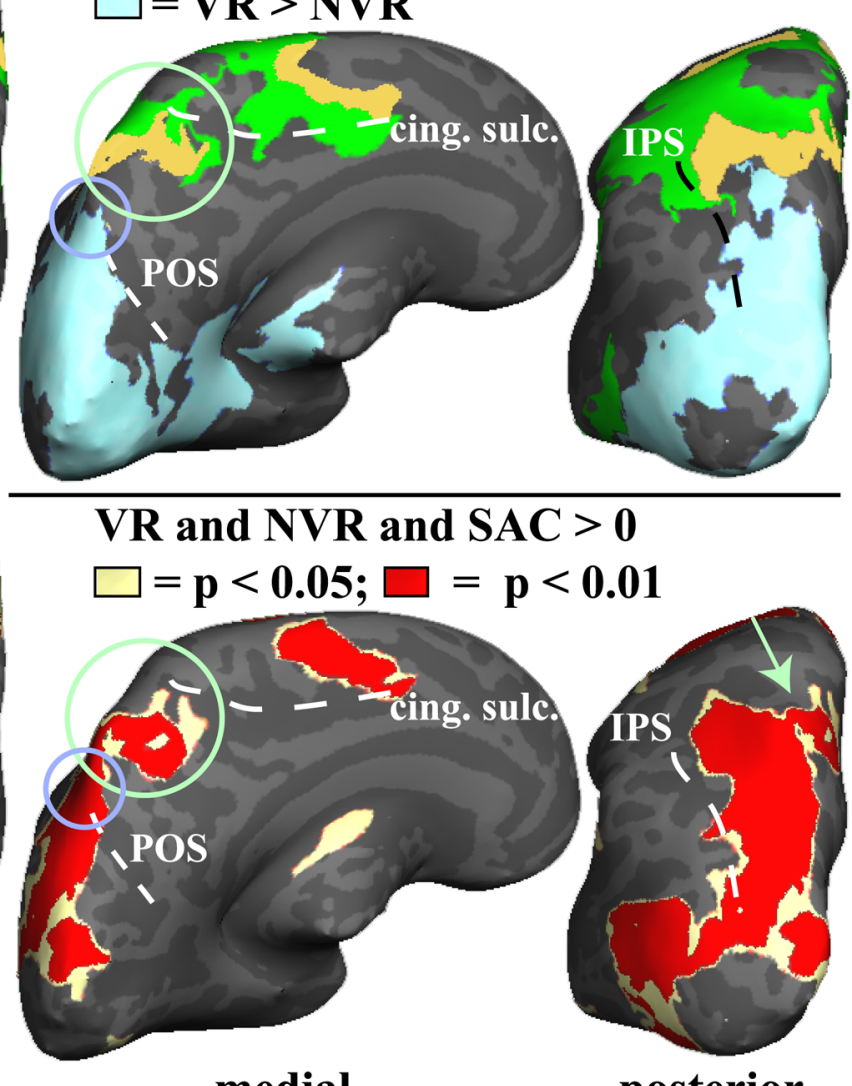

posterior

Figure 4. Conjunction analyses of group activations for visual reaching (VR), nonvisual reaching (NVR), and saccades (SAC). $\boldsymbol{A}$, Conjunction mask showing all the voxels (yellow) that participate in visual and nonvisual reaching as well as saccades, but more strongly in both reaches compared with saccades, and equally in both reaches. The left inflated hemisphere is shown. Only voxels are shown that satisfy all of the following conditions: (1) are significantly activated $(p<0.05)$ by each of the following: visual reach, nonvisual reach, and saccade compared with baseline; (2) are activated significantly more strongly ( $p<0.05)$ by visual reach than saccade and by nonvisual reach than saccade; and (3) are activated equally strongly by visual reach and nonvisual reach (visual reach - nonvisual reach $=0$, n.s.). Voxels that satisfy these requirements are found in the anterior precuneus (see green circle and arrows), as well as an area in medial intraparietal sulcus that extends dorsally, dorsal premotor cortex, and SMA. The circled area overlaps with the anterior precuneus activations shown in Figures 2 and 3. B, Overlay of multiple masks. Yellow, Conjunction mask from $A$. Green, All voxels that are significantly active in both visual and nonvisual reaches $(p<0.05)$ but are not significantly activated by saccades (n.s.). Blue, All voxels significantly $(p<0.05)$ more active in visual reach compared with nonvisual reach. Note the more anterior distribution of voxels exclusively activated by either kind of reaching (green), and not by able at www.jneurosci.org as supplemental material). Saccades were executed as in the first experiment. Both experiments show the same patterns of activation in the anterior precuneus and sPOS as reported here.

\section{Discussion}

We sought to identify sources of sensory input to human medial parietal reach regions during direct, immediately executed reaches that were either visible or invisible to subjects. We also compared visual and nonvisual direct reaching with saccades. Results show that an aPCu reachdominant region was activated equally with or without visual feedback from the reaching hand. aPCu was coactivated with medial IPS, and responded more to visual and nonvisual reaches than to saccades. This suggests a proprioceptive input from the moving arm in aPCu during the reach transport phase. A second, more posterior area at the superior POS responded more during visual than nonvisual reaches or saccades. sPOS may process visual feedback from the hand during on-line reaching or may calculate the visual distance between an effector and the target.

\section{Area aPCu}

Visual and nonvisual reaching activated aPCu equally. Reach-related activations were stronger in contralateral (left) aPCu, supporting a role for proprioception. This is consistent with left parietal damage deficits, e.g., optic ataxia and contralateral proprioceptive impairments (Perenin and Vighetto, 1988; Wolpert et al., 1998).

We always presented targets visually while manipulating the visual presence of

\footnotetext{
saccades, in parietal cortex; medial view shows that those activations fall mostly within the posterior cingulate sulcus. The small purple-blue circle shows that the superior POS is significantly more active during visual reaches than during nonvisual reaches. $\boldsymbol{C}$, Conjunction between all three conditions: voxels significantly activated by visual reaches, nonvisual reaches, and saccades, compared with baseline, are shown at $p<0.05$ (pale yellow) and, overlaid, at $p<0.01$ (in red). Note that this is a highly stringent test as voxels have to be active at $p<0.01$ in each condition versus baseline, as well as be active in all three conditions versus baseline. At higher thresholds, the medial (anterior precuneus) activation is becoming disconnected from the medial IPS and superior parietal cluster (see green arrows). The small purple-blue circle shows that the superior POS is significantly activated by all three conditions compared with baseline. All contrasts were performed voxelwise at the whole-brain level, with surfacebased clustering used for multiple-comparison correction (minimum cluster of $5 \times 5$ contiguous voxels). Note that "and" means the logical AND operator. CS, Central sulcus; cing. sulc., cingulate sulcus.
} 
L $\quad$ subject $1 \quad$ R

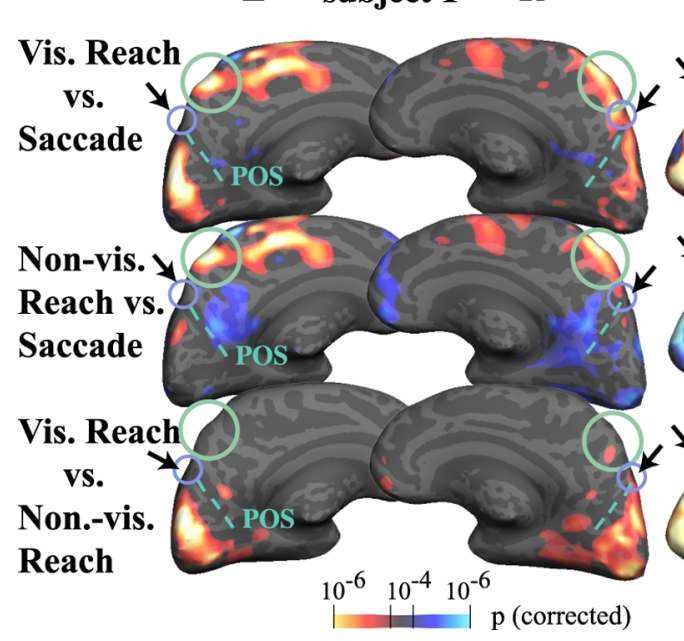

L $\quad$ subject 2

$\mathbf{R}$

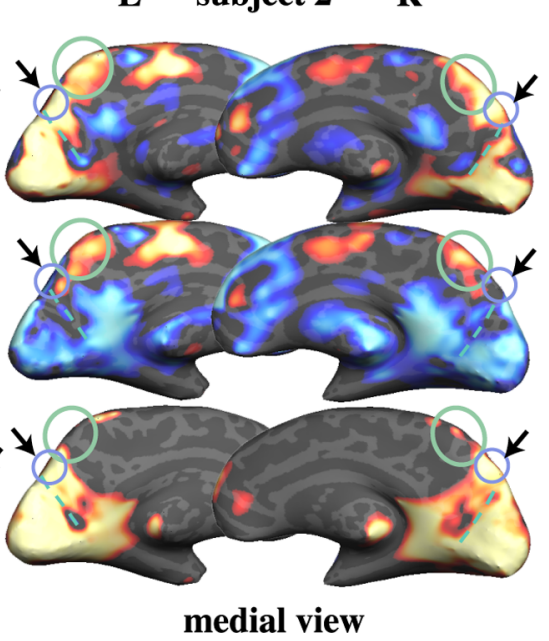

L $\quad$ subject $3 \quad$ R

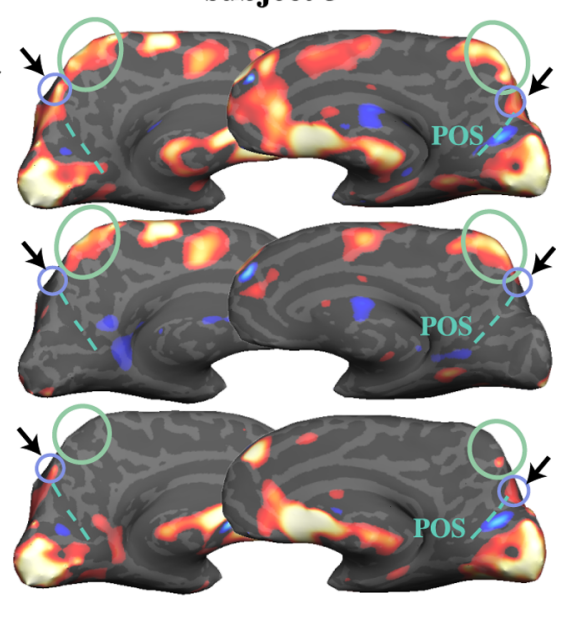

Figure 5. Individual data for visual direct reaching versus saccades, nonvisual direct reaching versus saccades, and visual versus nonvisual direct reaching. Differences in BOLD responses for the three conditions from three individual subjects. All subjects exhibited greater activations for either type of reach compared with saccades in aPCu (circled in green). Subjects showed variability in the location and amount of superior POS activation in the visual direct versus nonvisual direct reach comparison (see arrows and small purple-blue circles). Visual direct reaching compared with saccades (top row) activated the sPOS region more than nonvisual direct reaching compared with saccades (middle row) in seven of eight subjects. L, Left; $R$, right; vis., visual.

the hand. Common visual and nonvisual reaching activation in aPCu could partly be due to targets, rather than proprioceptive feedback. However, Pellijeff et al. (2006) found that eyes-closed reaching to nonvisual targets also activated aPCu. Subjects reached to point to the contralateral thumb or chin, with start and end points varying. aPCu activations were greater during the first reach following a postural change (in starting hand location or target location) than in subsequent reaches. Activations by postural changes without visual input strongly support proprioceptive inputs to aPCu. Non-spatially directed arm movements with eyes closed also show contralateral aPCu activation (Filimon, 2008). Thus, aPCu is activated without visual input. Interestingly, passive vibrotactile hand stimulation activates the precuneus (Beauchamp et al., 2007), perhaps by also stimulating muscle receptors.

Is aPCu purely somatosensory and not motor at all? Supplemental Figure S1 (available at www.jneurosci.org as supplemental material) shows strong aPCu activations during imagined reaches in the absence of proprioceptive input (see also Filimon et al., 2007). Imagined reaching did not activate S1, M1, or V1, suggesting that aPCu activations reflect motor preparation. This is consistent with previous studies showing hand movement planning without proprioceptive feedback activates aPCu (Astafiev et al., 2003).

Passive observation of reaching can also activate aPCu (Filimon et al., 2007). However, precuneus activations for both observation and imagery are weaker than for actual movement, suggesting that proprioception is important. aPCu activations also tended to be greater during motor imagery than observation, supporting a motor role. aPCu may have multiple populations of neurons, some visuomotor and some proprioceptive-motor. Nevertheless, our demonstration of equal aPCu activation for visual and nonvisual reaching suggests a dominant proprioceptive-motor representation.

The nomenclature of medial parietal cortex is not settled. Human aPCu, and possibly mIPS, may correspond to macaque PRR, which comprises parts of MIP and extends medially (Snyder et al., 1997; Andersen and Buneo, 2002), consistent with the present mIPS and aPCu coactivation. Alternatively, aPCu may represent macaque PEc. PEc is anterior to the POS and V6A, and responds during both passive joint rotations and reaching movements, supporting a role in proprioceptively directed reaching (Breveglieri et al., 2006). Human cytoarchitectonic research has identified anterior-posterior precuneus divisions resembling the present functional distinctions, proposing the terminology 7A and 7P/7M (Scheperjans et al., 2008).

Note that our aPCu activations are more dorsal than the precuneus activations frequently considered part of the "default network", which are frequently located on the inferior precuneus wall (Raichle and Snyder, 2007). Our results show that at least parts of the precuneus are actively involved in motor tasks, instead of being part of the "resting-state network." This is consistent with previously reported coordinates for hand movement activations [e.g., Connolly et al. (2003) and Hagler et al. (2007)].

\section{Superior POS}

More posteriorly located sPOS could represent both the location of the visual target relative to the visuomotor trajectory, and the visual distance from the hand or eye to the target. sPOS activations were stronger for visual reaching than nonvisual reaching or saccades, suggesting that sPOS is involved in visually monitoring how far the limb is from the target. However, nonvisual reaching also activated sPOS. This suggests the retinal target location is also represented in sPOS. Prado et al. (2005) found parietooccipital junction activations during nonvisual reaching to peripheral (as in the present study), not central, targets. The greater activation in sPOS with a visible hand supports a role in comparing the retinal locations of the hand and target.

Additional data (supplemental Fig. S1, available at www. jneurosci.org as supplemental material) show that sPOS has motor, not purely visual, properties (Filimon et al., 2007). Left sPOS responds strongly during imagined right-handed reaches, in the absence of visual input from the hand. Visual cortex, in contrast, is not activated relative to baseline, suggesting that sPOS activation is not due to visual imagery of a moving hand. Instead, the left sPOS activation indicates motor planning. It responds robustly during imagined and executed reaches, but more weakly during passive observation of a moving right hand. Thus, left sPOS seems to play a visuomotor, not just visual, role in reaching.

Pointing studies have revealed similar sPOS activations (Con- 
nolly et al., 2003). The sPOS may plan pointing movements by visual target location and visually perceived, rather than actual, movement direction (Fernandez-Ruiz et al., 2007). The sPOS was active when visually perceived pointing movements matched visual target locations, not muscle activations [e.g., when reversing prisms made leftward movements appear rightward (FernandezRuiz et al., 2007)], suggesting that sPOS encodes targets and movement plans in eye-centered coordinates. Also, sPOS prefers visual targets that can be acted on in near-space versus far-away targets (Quinlan and Culham, 2007; Culham et al., 2008), suggesting that sPOS contains visuomotor representations for action.

sPOS may be homologous to macaque V6A, where neurons are modulated by visual feedback during reaching (Fattori et al., 2001).

\section{Reaching versus pointing}

Filimon (2008) found stronger precuneus activations for arm versus finger movements; finger activations were shifted laterally. Finger pointing may not realistically approximate the reaching tasks used with macaques; thus, pointing-defined "PRRs" may differ from true (arm) parietal reach regions. Some pointing studies have labeled sPOS activations as PRR (Fernandez-Ruiz et al., 2007). Our greater aPCu activations for actual reaching versus saccades are consistent with studies that involved wrist (Astafiev et al., 2003) or forearm (Levy et al., 2007) movements. In contrast, studies of index finger movements found no aPCu activation differences between pointing and saccades (Hagler et al., 2007).

\section{Other regions}

Although we focused on the differential contributions of $\mathrm{aPCu}$ and sPOS to reaching versus saccades, additional parietal and frontal areas were also activated, consistent with previous reports on reaching (Filimon et al., 2007), reaching to grasp (Grafton et al., 1996; Culham et al., 2003), reaching to point or touch (Pellijeff et al., 2006; Levy et al., 2007), pointing (Connolly et al., 2000, 2003; Simon et al., 2002; Astafiev et al., 2003, 2004; Mendendorp et al., 2003; Fernandez-Ruiz et al., 2007; Hagler et al., 2007), and saccades (Culham et al., 1998; Connolly et al., 2000; Sereno et al., 2001; Koyama et al., 2004, Simon et al., 2002).

Posterior parietal regions more active for reaching than saccades included anterior IPS/supramarginal gyrus. These regions may represent human homologs of macaque AIP (Culham et al., 2003, 2006) and area 7b (Culham and Kanwisher, 2001), and were likely activated by hand-related rather than arm-related sensory stimulation during movement. Human homologs for macaque arm-movement areas have been proposed in medial parietal areas, however, which were the focus of the present study. Medial IPS was also coactivated with aPCu. It is possible that mIPS and aPCu form a functional unit. Other areas, e.g., the postcentral sulcus and the anterior superior parietal gyrus, likely correspond to macaque area 5. Our results show a gradual proprioceptive-to-visual transition from anterior to posterior parts of the posterior parietal lobule, from reach-only areas that do not respond to saccades in the very anterior parts of the posterior parietal lobule and at the posterior end of the cingulate sulcus, to both-reach-and-saccade areas in medial IPS and anterior precuneus, to more posterior reach areas modulated by vision of the hand and saccades (sPOS). This implies that even more functional subdivisions exist, supporting a network of multiple reach regions in humans.

\section{aPCu and attention}

Attention also activates the human precuneus (Beauchamp et al., 2001), consistent with attentional modulation in macaque parietal cortex (e.g., V6A, Galletti et al., 2003). However, attention is an unlikely explanation for our aPCu reaching activations. Covert (non-eye movement) attentional shifts activate the precuneus less than either saccades or pointing (Beauchamp et al., 2001; Astafiev et al., 2003). Here, overt saccades activated aPCu significantly less than reaching; thus, covert attention should activate aPCu even less. Finally, in additional tests we found that continuous arm, but not continuous finger or hand-only movements, activate aPCu with eyes closed, again ruling out greater attention to a continuously moving limb as the source of $\mathrm{aPCu}$ activation. Thus, the aPCu activations found here most likely reflect arm-movement execution rather than nonspecific spatial attention.

The term "parietal reach region" has been used loosely in the literature, with areas from the sPOS to the posterior cingulate sulcus all termed "PRR." Our results suggest that multiple, functionally distinct posterior parietal subregions participate in different aspects of reaching in humans.

\section{References}

Andersen RA, Buneo CA (2002) Intentional maps in posterior parietal cortex. Annu Rev Neurosci 25:189-220.

Astafiev SV, Shulman GL, Stanley CM, Snyder AZ, Van Essen DC, Corbetta M (2003) Functional organization of human intraparietal and frontal cortex for attending, looking, and pointing. J Neurosci 23:4689-4699.

Astafiev SV, Stanley CM, Shulman GL, Corbetta M (2004) Extrastriate body area in human occipital cortex responds to the performance of motor actions. Nat Neurosci 7:542-548.

Beauchamp MS, Petit L, Ellmore TM, Ingeholm J, Haxby JV (2001) A parametric fMRI study of overt and covert shifts of visuospatial attention. Neuroimage 14:310-321.

Beauchamp MS, Yasar NE, Kishan N, Ro T (2007) Human MST but not MT responds to tactile stimulation. J Neurosci 27:8261-8267.

Breveglieri R, Galletti C, Gamberini M, Passarelli L, Fattori P (2006) Somatosensory cells in area PEc of macaque posterior parietal cortex. J Neurosci 26:3679-3684.

Cavada C (2001) The visual parietal areas in the macaque monkey: current structural knowledge and ignorance. Neuroimage 14:S21-S26.

Connolly JD, Goodale MA, Desouza JFX, Menon RS, Vilis T (2000) A comparison of frontoparietal fMRI activation during anti-saccades and antipointing. J Neurophysiol 84:1645-1655.

Connolly JD, Andersen RA, Goodale MA (2003) fMRI evidence for a 'parietal reach region' in the human brain. Exp Brain Res 153:140-145.

Cox RW (1996) AFNI: software for analysis and visualization of functional magnetic neuroimages. Comput Biomed Res 29:162-173.

Culham JC, Kanwisher NG (2001) Neuroimaging of cognitive functions in human parietal cortex. Curr Opin Neurobiol 11:157-163.

Culham JC, Valyear KF (2006) Human parietal cortex in action. Curr Opin Neurobiol 16:205-212.

Culham JC, Brandt SA, Cavanagh P, Kanwisher NG, Dale AM, Tootell RB (1998) Cortical fMRI activation produced by attentive tracking of moving targets. J Neurophysiol 80:2657-2670.

Culham JC, Danckert SL, DeSouza JFX, Gati JS, Menon RS, Goodale MA (2003) Visually guided grasping produces fMRI activation in dorsal but not ventral stream areas. Exp Brain Res 153:180-189.

Culham JC, Cavina-Pratesi C, Singhal A (2006) The role of parietal cortex in visuomotor control: what have we learned from neuroimaging? Neuropsychologia 44:2668-2684.

Culham JC, Gallivan J, Cavina-Pratesi C, Quinlan DJ (2008) fMRI investigations of reaching and ego space in human superior parieto-occipital cortex. In: Embodiment, ego-space and action (Klatzky RL, Behrmann M, MacWhinney B, eds), pp 247-274. New York: Psychology.

Dale AM, Fischl B, Sereno MI (1999) Cortical surface-based analysis I: segmentation and surface reconstruction. Neuroimage 9:179-194.

Fattori P, Gamberini M, Kutz DF, Galletti C (2001) 'Arm-reaching' neurons 
in the parietal area V6A of the macaque monkey. Eur J Neurosci 13:2309-2313.

Fernandez-Ruiz J, Goltz HC, DeSouza JFX, Vilis T, Crawford JD (2007) Human parietal "reach region" primarily encodes extrinsic visual direction, not extrinsic movement direction, in a visual-motor dissociation task. Cereb Cortex 17:2283-2292.

Filimon F (2008) Non-spatial posterior parietal activations for finger, hand, and arm movements in humans. Soc Neurosci Abstr 34:854.16.

Filimon F, Nelson JD, Hagler DJ, Sereno MI (2007) Human cortical representations for reaching: mirror neurons for execution, observation, and imagery. Neuroimage 37:1315-1328.

Fischl B, Sereno MI, Dale AM (1999a) Cortical surface-based analysis II: inflation, flattening, and a surface-based coordinate system. Neuroimage 9:195-207.

Fischl B, Sereno MI, Tootell RB, Dale AM (1999b) High-resolution intersubject averaging and a coordinate system for the cortical surface. Hum Brain Mapp 8:272-284.

Galletti C, Kutz DF, Gamberini M, Breveglieri R, Fattori P (2003) Role of the medial parieto-occipital cortex in the control of reaching and grasping movements. Exp Brain Res 153:158-170.

Grafton ST, Fagg AH, Woods RP, Arbib MA (1996) Functional anatomy of pointing and grasping in humans. Cereb Cortex 6:226-237.

Hagler DJ Jr, Saygin AP, Sereno MI (2006) Smoothing and cluster thresholding for cortical surface-based group analysis of fMRI data. Neuroimage 33:1093-1103.

Hagler DJ Jr, Riecke L, Sereno MI (2007) Parietal and superior frontal visuospatial maps activated by pointing and saccades. Neuroimage 35:1562-1577.

Huang RS, Sereno MI (2008) Visual stimulus presentation using fiber optics in the MRI scanner. J Neurosci Methods 169:76-83.

Kalaska JF (1996) Parietal cortex area 5 and visuomotor behavior. Can J Physiol Pharmacol 74:483-498.

Koyama M, Hasegawa I, Osada T, Adachi Y, Nakahara K, Miyashita Y (2004) Functional magnetic resonance imaging of macaque monkeys performing visually guided saccade tasks: comparison of cortical eye fields with humans. Neuron 41:795-807.
Levy I, Schluppeck D, Heeger DJ, Glimcher PW (2007) Specificity of human cortical areas for reaches and saccades. J Neurosci 27:4687-4696.

Medendorp WP, Goltz HC, Vilis T, Crawford JD (2003) Gaze-centered updating of visual space in human parietal cortex. J Neurosci 23:6209-6214.

Pellijeff A, Bonilha L, Morgan PS, McKenzie K, Jackson SR (2006) Parietal updating of limb posture: an event-related fMRI study. Neuropsychologia 44:2685-2690.

Perenin MT, Vighetto A (1988) Optic ataxia: a specific disruption in visuomotor mechanisms. Different aspects of the deficit in reaching for objects. Brain 111:643-674.

Prado J, Clavagnier S, Otzenberger H, Scheiber C, Kennedy H, Perenin MT (2005) Two cortical systems for reaching in central and peripheral vision. Neuron 48:849-858.

Quinlan DJ, Culham JC (2007) fMRI reveals a preference for near viewing in the human parieto-occipital cortex. Neuroimage 36:167-187.

Raichle ME, Snyder AZ (2007) A default mode of brain function: a brief history of an evolving idea. Neuroimage 37:1083-1090; discussion 1097-1099.

Scheperjans F, Eickhoff SB, Hömke L, Mohlberg H, Hermann K, Amunts K, Zilles K (2008) Probabilistic maps, morphometry, and variability of cytoarchitectonic areas in the human superior parietal cortex. Cereb Cortex 18:2141-2157.

Sereno MI, Pitzalis S, Martinez A (2001) Mapping of contralateral space in retinotopic coordinates by a parietal cortical area in humans. Science 294:1350-1354.

Simon O, Mangin JF, Cohen L, Le Bihan D, Dehaene S (2002) Topographical layout of hand, eye, calculation, and language-related areas in the human parietal lobe. Neuron 33:475-487.

Snyder LH, Batista AP, Andersen RA (1997) Coding of intention in the posterior parietal cortex. Nature 386:167-170.

Ward BD (2000) Deconvolution analysis of fMRI time series data. AFNI $3 \mathrm{dDeconvolve} \mathrm{documentation,} \mathrm{Medical} \mathrm{College} \mathrm{of} \mathrm{Wisconsin.}$

Wolpert DM, Goodbody SJ, Husain M (1998) Maintaining internal representations: the role of the human superior parietal lobe. Nat Neurosci $1: 529-533$. 